\title{
Highlights of Cardiovascular Disease Studies Presented at the 2021 American Heart Association Scientific Sessions
}

\author{
Michelle T. Lee ${ }^{1,2} \cdot$ Jerin George ${ }^{3} \cdot$ Hunaina Shahab $^{4} \cdot$ Melody Hermel $^{5} \cdot$ Jamal S. Rana $^{6,7} \cdot$ Salim S. Virani ${ }^{1,3,8}$
}

Accepted: 1 December 2021 / Published online: 24 January 2022

This is a U.S. government work and not under copyright protection in the U.S.; foreign copyright protection may apply 2022

\begin{abstract}
Purpose of Review This review highlights major studies across a broad array of topics presented at the virtual 2021 American Heart Association (AHA) Scientific Sessions.

Recent Findings. Assessed studies examine a remotely delivered hypertension and lipid program in 10,000 patients across a diverse healthcare network; a cluster-randomized trial of a village doctor-led intervention for hypertension control; empagliflozin in heart failure with preserved ejection fraction (EMPEROR-Preserved); efficacy and safety of empagliflozin in hospitalized heart failure patients (EMPULSE); icosapent ethyl versus placebo in outpatients with coronavirus disease 2019 (PREPARE-IT 2); clinical safety, pharmacokinetics, and low-density lipoprotein cholesterol-lowering efficacy of MK-0161, an oral proprotein convertase subtilisin/kexin type 9 (PCSK9) inhibitor; and effects of aspirin on dementia and cognitive impairment in the ASCEND trial.

Summary Research presented at the 2021 AHA Scientific Sessions emphasized the importance of interventions for cardiovascular disease prevention.
\end{abstract}

Keywords Aspirin $\cdot$ Atherosclerotic cardiovascular disease $\cdot$ Cardiovascular prevention $\cdot$ Empagliflozin $\cdot$ Heart failure

\begin{tabular}{llll}
\multicolumn{2}{l}{ Abbreviations } & CAD & Coronary artery disease \\
ACS & Acute coronary syndrome & CI & Confidence interval \\
ACC & American College of Cardiology & COVID-19 & Coronavirus disease 2019 \\
AHA & American Heart Association & DBP & Diastolic blood pressure \\
ASCVD & Atherosclerotic Cardiovascular Disease & eGFR & Estimated glomerular filtration rate \\
BP & Blood pressure & HF & Heart failure
\end{tabular}

Salim S. Virani

virani@bcm.edu

Michelle T. Lee

Michelle.Lee@bcm.edu

Jerin George

Jerin.George@bcm.edu

Hunaina Shahab

Hunaina.Shahab@gmail.com

Melody Hermel

MelodyHermel@gmail.com

Jamal S. Rana

Jamal.S.Rana@kp.org

1 Health Policy, Michael E. DeBakey Veterans Affairs Medical Center Health Services Research \& Development Center (152), Quality \& Informatics Program2002 Holcombe Blvd, Houston, TX 77030, USA
2 Department of Medicine, Section of Health Services Research, Baylor College of Medicine, Houston, USA

3 Section of Cardiology, Department of Medicine, Baylor College of Medicine, Houston, TX, USA

4 Advanced Cardiac Imaging, Icahn School of Medicine at Mount Sinai, New York, NY, USA

5 Section of Cardiology, Scripps Clinic, La Jolla, CA, USA

6 Division of Cardiology, Kaiser Permanente Northern California, Oakland, CA, USA

7 Division of Research, Kaiser Permanente Northern California, Oakland, CA, USA

8 Section of Cardiology, Michael E. DeBakey Veterans Affairs Medical Center, Houston, TX, USA 


\begin{tabular}{|c|c|}
\hline HFE & Heart failure events \\
\hline HFmrEF & $\begin{array}{l}\text { Heart failure with mid-range ejection } \\
\text { fraction }\end{array}$ \\
\hline HFpEF & $\begin{array}{l}\text { Heart failure with preserved ejection } \\
\text { fraction }\end{array}$ \\
\hline HFrEF & Heart failure with reduced ejection fraction \\
\hline HHF & Hospitalization for heart failure \\
\hline HR & Hazard ratio \\
\hline HTN & Hypertension \\
\hline IPE & Icosapent ethyl \\
\hline KCCQ-TSS & $\begin{array}{l}\text { Kansas City Cardiomyopathy Questionnaire } \\
\text { Total Symptom Score }\end{array}$ \\
\hline LDL-C & Low-density lipoprotein cholesterol \\
\hline MI & Myocardial infarction \\
\hline OR & Odds ratio \\
\hline PCSK9 & Proprotein convertase subtilisin/kexin type 9 \\
\hline RRR & Relative risk reduction \\
\hline SBP & Systolic blood pressure \\
\hline SGLT-2 & Sodium-glucose cotransporter 2 \\
\hline TICSm & Telephone Interview for Cognitive Status \\
\hline VF & Verbal fluency \\
\hline
\end{tabular}

\section{Introduction}

The 2021 American Heart Association (AHA) Scientific Sessions featured several noteworthy clinical trials pertaining to cardiovascular disease (CVD) prevention and treatment. Reviewed studies assess a remotely delivered hypertension and lipid program in 10,000 patients across a diverse healthcare network; a cluster-randomized trial of a village doctor-led intervention; empagliflozin in heart failure with preserved ejection fraction (HFpEF; EMPEROR-Preserved) [1••]; icosapent ethyl (IPE) versus placebo in outpatients with coronavirus disease 2019 (COVID-19; PREPARE-IT 2); clinical safety, pharmacokinetics, and low-density lipoprotein cholesterol-lowering efficacy of MK-0161, an oral proprotein convertase subtilisin/kexin type 9 (PCSK9) inhibitor; and effects of aspirin on dementia and cognitive impairment in the ASCEND trial. Like prior publications, we will discuss the significance and clinical implications of select research presentations [2-4]. A table summarizing the studies discussed is included at the end of this manuscript (Table 1).

\section{A remotely delivered hypertension and lipid program in 10,000 patients across a diverse healthcare network}

\section{Study Overview}

Disparities in cardiovascular disease management are apparent in the undertreatment of hypertension (HTN) and hypercholesterolemia [5-7]. Despite available treatment options, up to half of the patients with modifiable cardiovascular risk factors do not receive adequate medical care $[8,9]$. To overcome this clinical challenge, a focus on remote health has emerged. Still, there is concern regarding the "digital health divide" whereby those who are less likely to connect to healthcare via digital means experience a barrier to care which may exacerbate health inequities [10-13]. The Digital Care Transformation Study regarding lipid and hypertension control was designed to address these gaps in care by means of a remote program.

Within the Mass General Brigham health system, patients in need of HTN or low-density lipoprotein cholesterol (LDL-C) optimization were identified by provider referral and electronic health record screening. A remote care delivery platform was enabled by a team of nonlicensed navigators and pharmacists who gathered data and provided education, prescriptions based on clinical algorithms, and communicated decisions remotely. HTN management was enabled by WiFi, cellular, and Bluetooth devices. Personalized remote care was delivered without in-person visits with physicians. Program oversight was provided by specialists and primary care physicians. Outcomes of interest included reductions in blood pressure (BP) and LDL-C from baseline to completion of the program.

Enrollment totaled 11,000 patients of whom $12 \%$ were $>75$ years of age, $55 \%$ were female, $29 \%$ were nonWhite, and $8 \%$ were non-English speaking. Twenty-nine percent had established atherosclerotic cardiovascular disease (ASCVD), 22\% had diabetes without ASCVD, $26 \%$ had LDL $>190$ without diabetes or ASCVD, and $23 \%$ were deemed high-risk primary prevention. The mean change in BP from program baseline to exit was a change of $10 / 6 \mathrm{mmHg}$ for all enrolled patients and $12 / 6 \mathrm{mmHg}$ in patients who completed the program and achieved maintenance. Ninety-two percent of patients who completed the program reached their guidelinerecommended $\mathrm{BP}$ goals as defined by the ACC/AHA in this patient population as a $\mathrm{BP}$ target of $<130 / 80 \mathrm{mmHg}$. Significant reductions in LDL-C were noted in all enrolled patients, $45 \mathrm{mg} / \mathrm{dL}$ change in cholesterol, with even more pronounced reduction in LDL-C noted in those who completed the program, $70 \mathrm{mg} / \mathrm{dL}$ representing a $50 \%$ drop from baseline. Ninety-four percent of patients who completed the program achieved their LDL-C guideline-directed goals as determined by the ACC/AHA guidelines. Benefits remained consistent when analyzed across race, ethnicity, and primary language subgroups. Engagement and retention were also similar across each of these subgroups. 


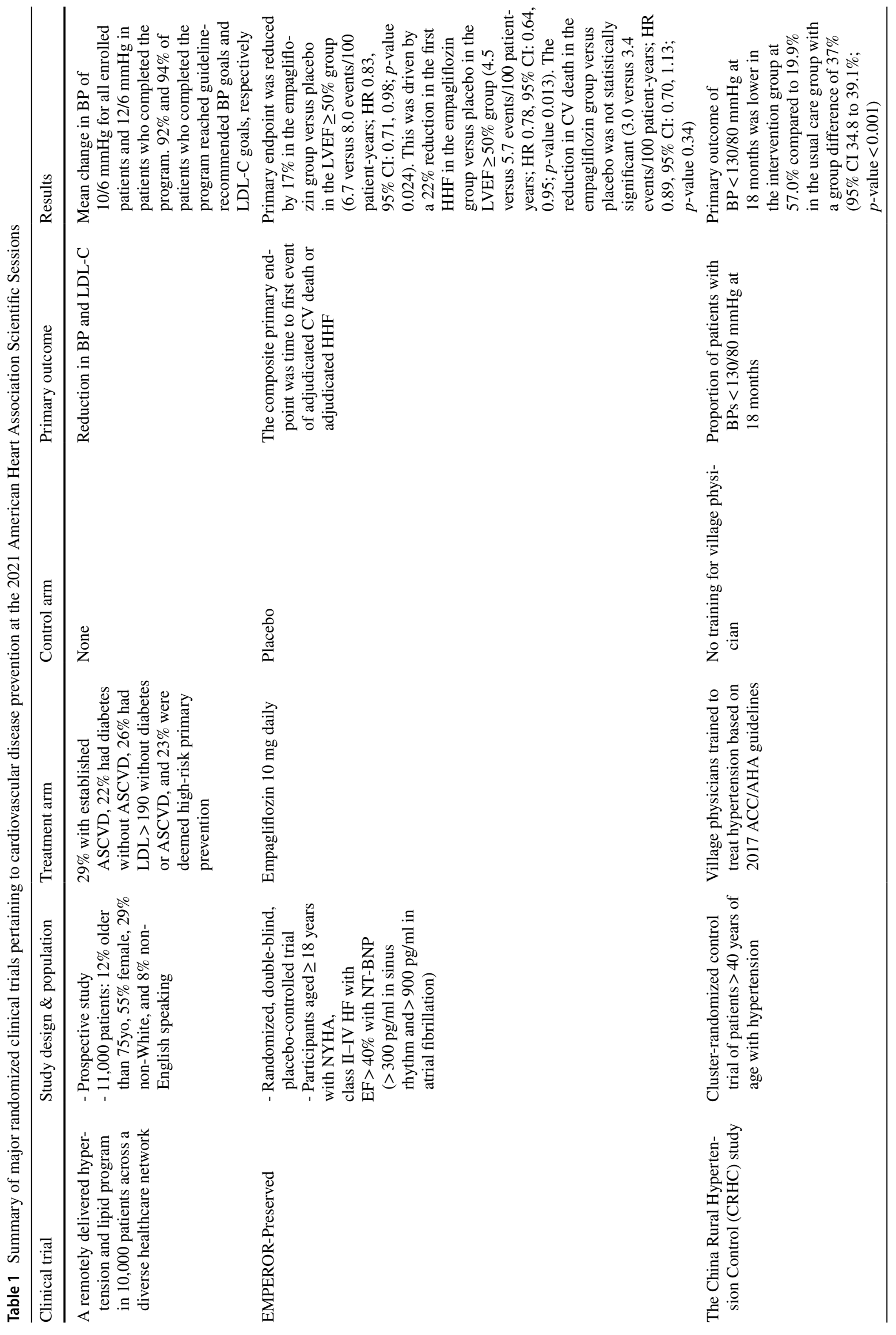




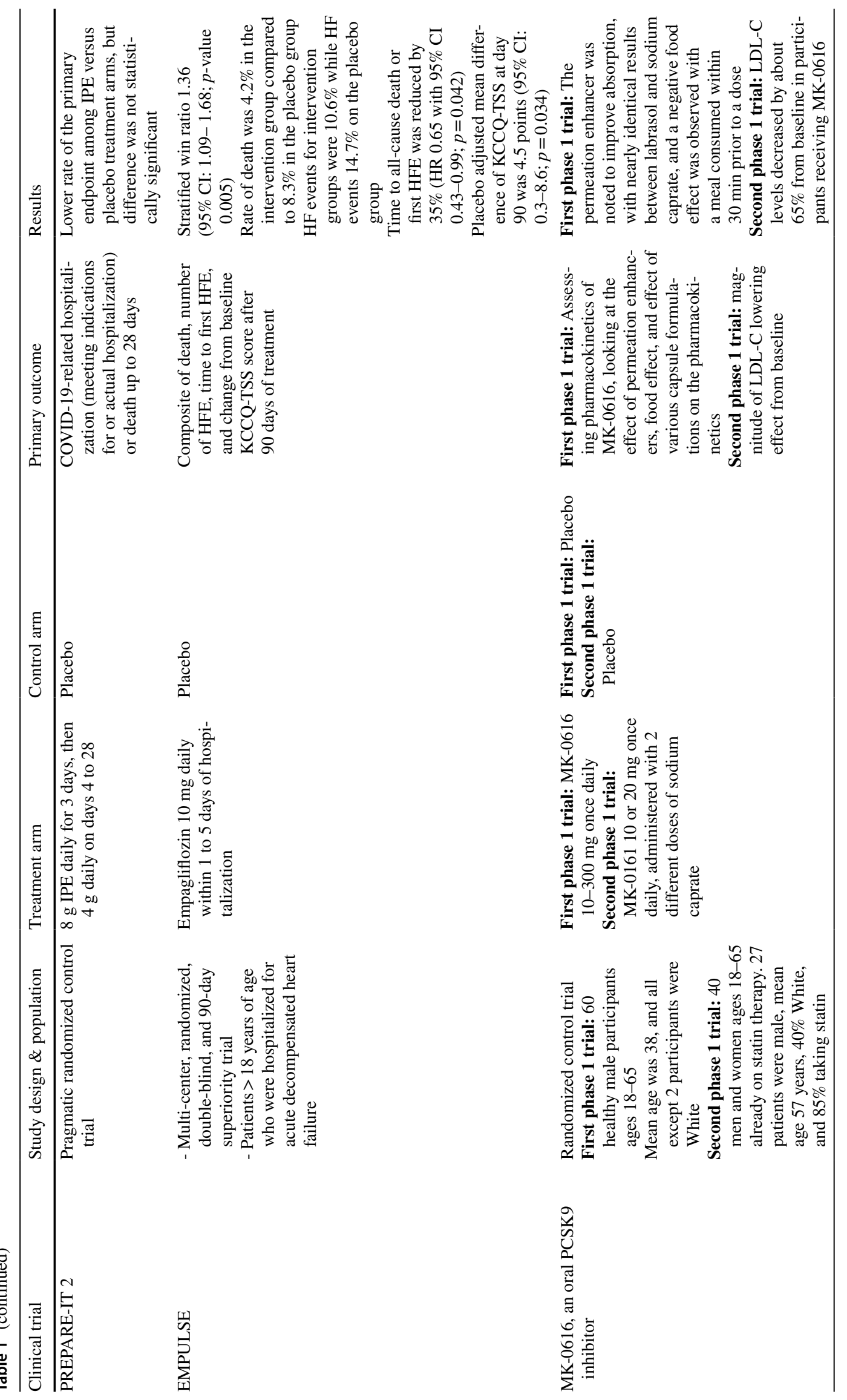




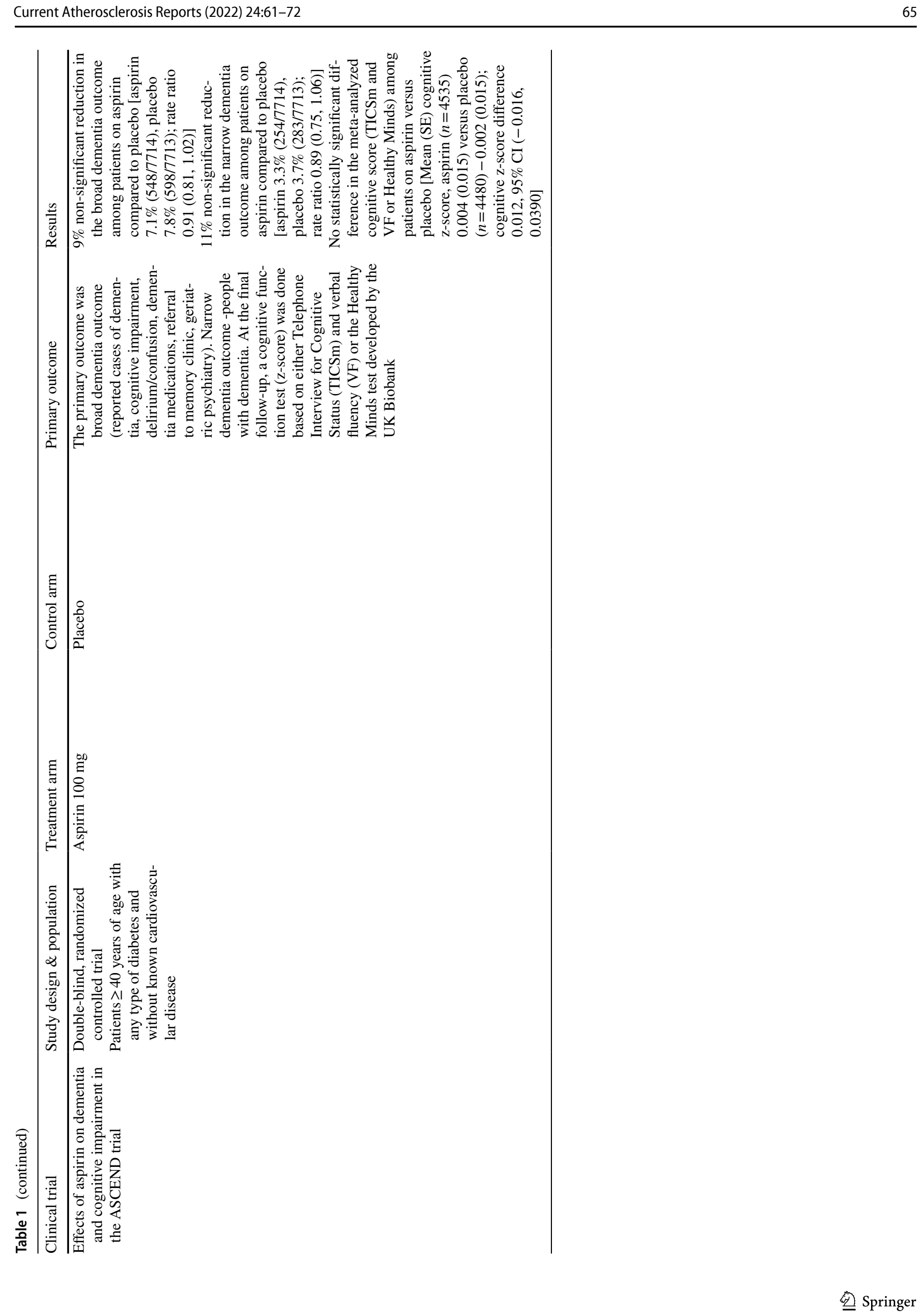




\section{Clinical Implication}

In the modern era, undertreatment of hypertension and hypercholesterolemia remains a persistent problem. The present trial, representing a diverse patient cohort, demonstrated clinically meaningful reductions in BP and LDL-C through remote patient care. Even small changes in $\mathrm{BP}(1-2 \mathrm{mmHg})$ have been shown to have a significant impact on population rates of cardiovascular disease [14•], and this trial demonstrates a remarkable mean change in BP of $10 / 6 \mathrm{mHg}$ for all enrolled participants. Moreover, LDL-C was lowered by $45 \mathrm{mg} / \mathrm{dL}$ in all enrolled participants. This reduction in these two extremely prevalent and important cardiovascular risk factors can translate into fewer ASCVD events. Remote patient care remains a very attractive option especially in the era of the COVID-19 pandemic. The incorporation of patient navigators enabled remote patient contact and education thereby expanding access to care. The algorithmically directed care supported by physicians and managed by pharmacists allowed for automated workflows, streamlined communication, and tailored medical therapy to reach guideline-directed targets. This system design and remote care delivery platform is timely and of crucial importance to practically apply management guidelines and improve patient care.

While impressive results are noted for those patients who completed the program, it is important to note that most patients in both the HTN and lipid program became unreachable over the course of the program. Still, engagement and retention were similar across race, ethnicity, and primary language subgroups. Future studies will need to continue to optimize techniques to keep patients actively engaged in similar remote programs without being lost to follow-up.

\section{A cluster-randomized trial of a village doctor-led intervention}

\section{Study Overview}

The China Rural Hypertension Control (CRHC) study is a cluster-randomized trial to evaluate the effectiveness of a standard treatment protocol based on the 2017 American College of Cardiology/American Heart Association (ACC/AHA) hypertension treatment guidelines on hypertension control and cardiovascular events. The investigators selected 326 villages from 3 provinces in China which included a total of 33,995 participants aged $\geq 40$ years with an untreated $\mathrm{BP} \geq 140 / 90 \mathrm{~mm} \mathrm{Hg}$ or treated $\mathrm{BP} \geq 130 / 80 \mathrm{~mm} \mathrm{Hg}$ or with an untreated $\mathrm{BP} \geq 130 / 80 \mathrm{~mm} \mathrm{Hg}$ and a history of cardiovascular disease, diabetes mellitus, or chronic kidney disease. The villages were randomly assigned to a treatment group where the physicians were trained to manage hypertension (163 villages) or usual management group (163 villages). In the treatment group, the village physicians were trained on standard BP measurement, received support from other physicians (primary care physicians and hypertension specialists), and received performance-based financial incentives. The enrolled patients in the treatment group received health coaching on home BP monitoring, lifestyle changes, and adherence to medication as well as discounted or free antihypertensive medication. The primary outcome measure was the proportion of patients with BPs $<130 / 80 \mathrm{mmHg}$ at 18 months. The secondary outcomes were the proportion of patients with $\mathrm{BP}<140 / 80 \mathrm{mmHg}$ at 18 months and change in systolic and diastolic BP from baseline to 18 months. Intention to treat analysis was conducted.

The primary outcome of the proportion of patients with $\mathrm{BP}<130 / 80 \mathrm{mmHg}$ at 18 months was lower in the intervention group at $57.0 \%$ compared to $19.9 \%$ in the usual care group with a group difference of $37 \%$ (95\% CI 34.8 to $39.1 \%$; $p$-value $<0.001$ ). Secondary outcomes including change in BP from baseline to 18 months was $-26.3 /-14.6 \mathrm{mmHg}$ (systolic/diastolic) in the intervention group compared to $-11.8 /-7.5 \mathrm{mmHg}$ (systolic/diastolic) in the usual care group with a net difference of $-14.5 /-7.1 \mathrm{mmHg}$ (95\% CI: -15.7 to $-13.3 \mathrm{mmHg} ; p$-value $<0.001$ for $\mathrm{SBP}$ and $95 \%$ CI: -7.7 to $-6.5 \mathrm{mmHg}$; $p$-value $<0.001$ for DBP). The proportion of patients with $\mathrm{BP}<140 / 90 \mathrm{mmHg}$ at 18 months was $77.3 \%$ for the intervention group compared to $44.5 \%$ in the usual care group ( $p$-value $<0.001$ ). The results were consistent by age, sex, education, and percent of anti-hypertensive medication use.

\section{Clinical Implication}

The prevalence of hypertension in China is high and increasing. A recent national survey in 2014 showed $27.8 \%$ of Chinese adults with hypertension, and only $5.5 \%$ of hypertensive patients in rural China had their BP controlled. Village doctors with appropriate training could play an important role in hypertension control in rural China. This trial showed a significant decrease in BP which was sustained at 18 months. This implementation strategy could be scaled up in rural China and other low resource settings for hypertension control which can overall help improve cardiovascular disease burden. 


\section{EMPEROR-Preserved: empagliflozin in heart failure with preserved ejection fraction (HFpEF) patients}

\section{Study Overview}

The EMPEROR-Preserved trial previously showed that empagliflozin, a sodium-glucose cotransporter 2 (SGLT2) inhibitor, reduced the combined risk of cardiovascular (CV) death and heart failure (HF) hospitalizations among patients with HF with left ventricular ejection fraction $(\mathrm{LVEF})>40 \%$ regardless of diabetes status $[1 \bullet \bullet]$. In this current study, these results were further stratified by baseline LVEF $\geq 50 \%$ (true HFpEF) versus LVEF 41 to $49 \%$ (heart failure with mid-range ejection fraction; HFmrEF). The aim was to assess the effects of empagliflozin, in addition to standard therapy, in patients with LVEF $\geq 50 \%$ in the EMPEROR-Preserved trial versus patients with LVEF $41-49 \%$ and to compare them to the other relevant trial results. This phase 3, double-blind, placebo-controlled trial recruited a total of 5988 patients, aged $\geq 18$ years, with class II to IV heart failure (HF), with or without type 2 DM with NT-BNP (>300 pg/ml in sinus rhythm and $>900 \mathrm{pg} / \mathrm{ml}$ in atrial fibrillation). Participants were randomized in a 1:1 fashion to receive either empagliflozin $10 \mathrm{mg}(n=2997)$ or matching placebo $(n=2991)$. The composite primary endpoint was time to first event of adjudicated $\mathrm{CV}$ death or adjudicated hospitalization for heart failure (HHF). The secondary endpoints were the first and recurrent adjudicated HHF events and the slope of change in estimated glomerular filtration rate (eGFR).

The mean age of LVEF $\geq 50 \%$ group $(n=4005 ; 67 \%)$ was 72.8 years whereas that of the LVEF $41-49 \%$ group $(n=1983 ; 33 \%)$ was 70.1 years $(p<0.001)$. There were $50 \%(n=2019)$ women in the LVEF $\geq 50 \%$ group whereas the LVEF $41-49 \%$ group had $33 \%(n=657)$ women $(p<0.001)$. Both the groups had around $50 \%$ patients with diabetes (48\% in HFpEF, $52 \%$ in HFmrEF; $p=0.004$ ). The standard HF therapy for both the groups included ACE inhibitors/angiotensin receptor blockers/angiotensin receptor-neprilysin inhibitors (79\% in HFpEF, $85 \%$ in HFmrEF, $p$-value 0.001 ), beta blocker $(84 \%$ in $\mathrm{HFpEF}, 90 \%$ in HFmrEF, $p<0.001$ ), mineralocorticoid antagonist (33\% HFpEF, $47 \%$ in HFmrEF, $p$-value $<0.001$ ), and diuretics ( $81 \%$ in HFpEF, 79\% in HFmrEF, $p$-value 0.041). The primary endpoint was reduced by $17 \%$ in the empagliflozin group versus placebo in the $L V E F \geq 50 \%$ group (6.7 versus 8.0 events/100 patient-years; hazard ratio [HR] $0.83,95 \%$ confidence interval [CI]: 0.71-0.98; $p$-value 0.024$)$. This was driven by a $22 \%$ reduction in the first HHF in the empagliflozin group versus placebo in the LVEF $\geq 50 \%$ group (4.5 versus 5.7 events/100 patient-years; HR 0.78,
95\% CI: 0.64-0.95; p-value 0.013). The reduction in CV death in the empagliflozin group versus placebo was not statistically significant (3.0 versus 3.4 events/100 patientyears; HR 0.89, 95\% CI: 0.70, 1.13; $p$-value 0.34). Similarly, the change in all-cause mortality in empagliflozin versus placebo (6.1 versus 6.1 events/100 patient-years; HR 1.02 , 95\% CI: $0.86-1.21 ; p=0.84$ ) and total HHF (6.8 versus 7.9 events/100 patient-years; HR $0.83,95 \%$ CI: $0.66-1.04 ; p=0.11$ ) in the $\mathrm{LVEF} \geq 50 \%$ group was not statistically significant.

In the HFmrEF patients with LVEF 41-49\% group receiving empagliflozin versus placebo, the primary endpoint was reduced by $29 \%$ (7.2 versus 10 events/100 patient-years; HR $0.71,95 \%$ CI: $0.57-0.88 ; p=0.002 ; p$ for interaction 0.27 ), driven by a statistically significant reduction in first HHF of $42 \%$ (3.8 versus 6.5 events/100 patient-years; HR 0.58, 95\% CI: $0.44-0.77 ; p<0.001 ; p$ for interaction 0.093 ). Total HHF in the LVEF $41-49 \%$ group was reduced by $43 \%$ in the empagliflozin group versus placebo (5.8 versus 10.1 events/100 patient-years; HR 0.57, 95\% CI: 0.42-0.79; $p$-value $<0.001$ ) ( $p$ for interaction 0.06 ). The $8 \%$ reduction in $\mathrm{CV}$ death and $4 \%$ reduction in all-cause mortality in the LVEF 41-49\% group were not statistically significant (HR $0.92,95 \%$ CI: $0.69-1.22 ; p=0.54$ and HR $0.96,95 \%$ CI: $0.78-1.19$; $p$-value 0.72 , respectively). At week 52 , the change from baseline in Kansas City Cardiomyopathy Questionnaire Clinical Summary Score (KCCQ-CSS) quality of life in the empagliflozin versus placebo in the $\mathrm{LVEF} \geq 50 \%$ group was 4.24 versus $2.78(p=0.006)$, while in LVEF $41-49 \%$ group, it was 4.86 versus 3.3 ( $p=0.043 ; p$-value for interaction 0.92). Similarly, there was a significant improvement in KCCQ total score and overall summary score in all patients who received empagliflozin versus placebo.

When patients with LVEF $>50 \%$ in the EMPEROR-Preserved trial $(n=3501)$ were compared to similar patients in the PARAGON-HF trial $(n=4067)$ [9], patients in the EMPEROR-Preserved trial had a significant reduction in first HHF or CV death (HR 0.82; 95\% CI 0.69-0.98, $p=0.0263$ versus HR $0.94 ; 95 \%$ CI $0.82-1.08, p=0.38$ ). When compared to placebo, the difference in the slope of decline in GFR with empagliflozin in the LVEF $\geq 50 \%$ group over time was $1.24 \mathrm{ml} / \mathrm{min} / 1.73 \mathrm{~m}^{2}$ per year $(p<0.0001)$.

\section{Clinical Implication}

The EMPEROR-Preserved trial demonstrates the superiority of empagliflozin over placebo in the reduction of composite endpoint of the first event of CV death or HF hospitalization patients with LVEF $\geq 50 \%$. The benefit was primarily driven by a reduction in HF hospitalization-related events and not mortality. These benefits appeared irrespective of the baseline EF. This is the first large-scale study to demonstrate 
significant improvement in patients with true HFpEF with drug therapy.

\section{EMPULSE: efficacy and safety of empagliflozin in hospitalized heart failure patients}

\section{Study Overview}

The purpose of this study was to assess the clinical benefit, safety, and tolerability of empagliflozin on hospitalized patients for acute decompensated heart failure. This was a multi-center, randomized, double-blind, and 90-day superiority trial. Inclusion criteria included patients over 18 years of age who were currently hospitalized for a primary diagnosis of acute decompensated heart failure (regardless of ejection fraction or a diagnosis type 2 diabetes mellitus), elevated NT-proBNP $>1600 \mathrm{pg} / \mathrm{mL}$ or BNP $>400 \mathrm{pg} / \mathrm{mL}$, and with treatment of intravenous diuretics such as $40 \mathrm{mg}$ of furosemide or equivalent. Inclusion criteria also included clinical stability with systolic $\mathrm{BP}>100 \mathrm{mmHg}$, no increase in intravenous diuretic dose prior to randomization, no intravenous vasodilators, and no intravenous inotropic drugs. Key exclusion criteria included cardiogenic shock, current hospitalization for acute heart failure from an acute myocardial infarction, pulmonary embolism, or cerebrovascular accident. Interventions such as major cardiac procedures such as percutaneous coronary intervention transcatheter aortic valve intervention, MitraClip placement, cardiac resynchronization therapy, or cardiac mechanical support implantation. Patients with eGFR $<20 \mathrm{~mL} / \mathrm{min} / 1.73 \mathrm{~m}^{2}$ or requiring dialysis, type 1 diabetics, or history of ketoacidosis were excluded from the study as well. Patients were randomized to the control arm or treatment arm with empagliflozin $10 \mathrm{mg}$ greater than $24 \mathrm{~h}$ and less than 5 days of hospitalization.

The primary outcome measure was a clinical benefit which was composite of death, number of heart failure events (HFE), time to first HFE, and change from baseline Kansas City Cardiomyopathy Questionnaire Total Symptom Score (KCCQ-TSS) after 90 days of treatment. Baseline characteristics of the intervention group and placebo group were similar with an average age of the patients around 70 years of age. One-third of the patients had LVEF $>40 \%$, one-third had LVEF $\leq 40 \%$, and one-third admitted for de novo HF. The primary analysis was assessed by a stratified win ratio. The win ratio was performed by a computer as it randomly picked one patient from the placebo group and one patient from the treatment group. It then checks if a patient died and if death was in the placebo group, then the patient in the treatment group had won. If there was a tie, the computer would then check the frequency of HHF. A tie in this category would lead the computer to pick time to first HFE and then another tie would lead the computer to evaluate the KCCQ-TSS score.

The stratified win ratio was 1.36 (95\% CI: 1.09-1.68; $p$-value 0.0054$)$ and death was $4.2 \%$ in the intervention group compared to $8.3 \%$ in the placebo group. HFE for intervention groups were $10.6 \%$ while HF events were $14.7 \%$ in the placebo group. Primary endpoint by subgroup analysis also showed consistent effects of empagliflozin including among those with de novo HF, acute on chronic HF, with lower or higher ejection fraction than $40 \%$, or with or without diabetes mellitus. Time to all-cause death or first HFE was reduced by $35 \%$ (HR $0.65,95 \%$ CI $0.4-0.99$; $p=0.042$ ). Placebo adjusted mean difference of KCCQ-TSS at day 90 was 4.5 points (95\% CI $0.3-8.6 ; p=0.0347$ ). Body weight change from baseline to day 90 was a reduction by $1.5 \mathrm{~kg}$ in the intervention group compared to placebo $(95 \% \mathrm{CI}-2.8$ to $-0.3 ; p=0.0137)$. Adverse events that took place were similar in the intervention and placebo groups. Patients treated with empagliflozin during their hospitalization were $36 \%$ more likely to experience a clinical benefit compared to patients on placebo.

\section{Clinical Implication}

Over the last few years, SGLT-2 inhibitors have shown benefit in patients with HF irrespective of ejection fraction or diabetes status. The EMPULSE trial adds to the increasing body of evidence in SGTL-2 inhibitors in HF therapy as it was the first trial to test inpatients with decompensated HF regardless of ejection fraction or diabetes mellitus status. Initiation of empagliflozin versus placebo in patients hospitalized for acute HF resulted in a significant clinical benefit within 90 days, fewer deaths or HFE, improvement in quality of life, greater reduction in body weight, and benign safety profile.

\section{PREPARE-IT 2: icosapent ethyl (IPE) versus placebo in outpatients with COVID-19}

\section{Study Overview}

PREPARE-IT 2 is a pragmatic trial assessing IPE versus placebo in non-hospitalized patients with COVID19 to reduce hospitalization rates and major clinical complications. Included participants were $\geq 40$ years of age with confirmed COVID-19 diagnosis within 7 days of symptom onset. Exclusion criteria encompassed hospitalization requirement, contraindication to treatment drug, pregnant or breastfeeding women, anticoagulant administration, or hemorrhagic diathesis. Patients in the treatment arm received $8 \mathrm{~g}$ of IPE daily for the first 
3 days followed by $4 \mathrm{~g}$ daily on days four to 28 . Those randomized to the placebo arm received a placebo capsule with identical dosing schedules as the active group. The primary study outcome was COVID-19-related hospitalization or death up to 28 days. For the primary outcome, hospitalization entailed meeting indications for inpatient admission per the blinded investigator or actual hospitalization. The key secondary outcomes included COVID-19-related actual hospitalization or death assessed up to 28 days. Other secondary endpoints entailed not alive or not out of the hospital at day 28 , new requirement of mechanical ventilation, total evens (non-fatal myocardial infarction [MI] or non-fatal stroke or death), and total mortality. Investigators calculated a sample size of 2000 participants based on $90 \%$ power to detect a $30 \%$ relative risk reduction (RRR) in the active group if the 28-day composite rate (indication for hospitalization or death) among controls was $18 \%$, assuming a $5 \%$ dropout and total significance alpha level of 0.05 .

Of the 2052 patients included in the study, approximately $53 \%$ were female and the average age was 53 years. The most commonly reported symptoms were fatigue, cough, and fever. The primary outcome was experienced by 110 of 986 patients in the treatment arm (11.16\%) and 135 of 1030 patients in the placebo arm (13.69\%) without significant difference noted between the two groups (HR 0.84, 95\% CI $0.65-1.08 ; p=0.17)$. Similarly, there was no significant difference related to the key secondary outcome among the 53 of 986 patients in the treatment arm $(5.38 \%)$ and 70 of 1030 patients in the placebo arm $(6.80 \%$; HR 0.78 , 95\% CI $0.55-1.12 ; p=0.18)$. Other secondary endpoints measured also did not show significant differences between the treatment versus placebo arms: not alive or not out of the hospital at day 28 ( 10 vs. 14 patients; odds ratio [OR] $0.74,95 \%$ CI $0.29-1.81 ; p=0.54)$, new requirement of mechanical ventilation ( 8 vs. 11 patients; OR $0.76,95 \%$ CI $0.26-2.08 ; p=0.65$ ), non-fatal myocardial infarction MI or non-fatal stroke or death (4 vs. 11 patients; OR $0.38,95 \%$ CI $0.09-1.28 ; p=0.12$ ), total mortality (4 vs. 8 patients; OR $0.52,95 \%$ CI $0.11-1.95 ; p=0.39$ ), and in-hospital length of stay ( 9 vs. 7 days; mean difference 3 days, 95\% CI 1-5; $p=0.01$ ). Regarding safety, IPE was well-tolerated without significant differences between the active and placebo arms. In terms of drug discontinuation rate, there was a significant difference seen among active treatment (70 of 989 patients [7.08\%]) versus placebo (39 of 1030 [3.79\%]) groups $(p=0.001)$. Statistically significant reasons for such stopping the study included patient discontinuation (active: 25 of 989 patients [2.43\%], placebo: 12 of 1030 patients [1.21\%]; $p=0.030$ ) and both adverse event and patient discontinuation (active: 16 of 989 patients [1.55\%], placebo: 3 of 1030 patients [0.30\%]; $p=0.002$ ).

\section{Clinical Implication}

Overall, despite lower observed rates of COVID-19-related hospitalization and death among patients in the IPE treatment versus placebo arm, no statistically significant difference was seen between the two groups. This trend of lower rates and odds remained consistent for other measured secondary endpoints. However, both primary and key secondary endpoints did not reach statistical significance likely due to the underpowered study. Additionally, there was a slightly higher discontinuation rate among those in the active treatment arm even though IPE was generally well-tolerated without significant differences in reported adverse events when compared to placebo. Future investigations with a larger patient population to achieve higher statistical power are needed to determine if IPE has a role in COVID-19 management.

\section{The clinical safety, pharmacokinetics, and LDL-C-lowering efficacy of MK-0616 an oral PCSK9 inhibitor}

\section{Study Overview}

Despite available therapies directed at cholesterol lowering, millions of patients with hypercholesterolemia do not achieve LDL-C treatment targets. The addition of proprotein convertase subtilisin/kexin type 9 (PCSK9) inhibition to statin therapy decreases LDL-C by at least an additional $50 \%$, making it possible to achieve very low LDL-C levels and decrease ASCVD events $[15,16]$. Currently available PCSK9 inhibitors, alirocumab and evolocumab, are underused due to cost, insurance authorization, and the need for biweekly or monthly injections. Still, their clinical utility for high-risk patients in need of aggressive secondary prevention or those with familial hypercholesterolemia has been powerful [17]. Currently, there are no orally bioavailable PCSK9 inhibitors given the difficulty to find small molecules that can disrupt the interaction of LDL receptors and the PCSK9 protein.

This study included two small phase 1 randomized double-blind placebo-controlled trials looking at dosage, safety, and pharmacokinetics of MK-0616 used in conjunction with agents to improve gastrointestinal absorption. The first trial involved a population of 60 healthy male participants ages $18-65$ who were randomized to receive once-daily doses ranging from $10 \mathrm{up}$ to $300 \mathrm{mg}$ of MK-0616 or placebo. The mean age was 38 years, and all except 2 participants were White. Outcomes of interest included assessing pharmacokinetics of MK-0616, looking at the effect of permeation enhancers, food effect, and effect of various capsule formulations on the 
pharmacokinetics. Percent change in free PCSK9 in the plasma was measured. Patients were monitored for any serious side effects. The second trial involved the addition of MK-0616 to the treatment regimen of 40 men and women ages 18-65 with hypercholesterolemia already on moderate- to high-intensity statin therapy for at least 3 months. These patients were studied for 14 days with the primary outcome of interest being the magnitude of LDL-C-lowering effect from baseline. Twenty-seven patients were male, mean age was $57,40 \%$ of patients were White, and $85 \%$ of patients were taking moderate- to high-intensity statin. Patients were randomized to receive once-daily dose of 10 or $20 \mathrm{mg}$ of MK-0616 administered with 2 different doses of sodium caprate versus placebo and with meal.

Results from the first trial demonstrated no deaths or serious adverse events. Treatment-related adverse events were mild. These included abdominal discomfort, diarrhea, dyspepsia, headache, and maculopapular rash. The permeation enhancer was noted to improve absorption, with nearly identical results between labrasol and sodium caprate, and a negative food effect was observed with a meal consumed within $30 \mathrm{~min}$ prior to a dose. Of the studied dosages, there was more than a $90 \%$ maximal reduction of free PCSK9 in the plasma for $24 \mathrm{~h}$ despite only $2 \%$ oral bioavailability. Similarly, in the second trial, there were no deaths or serious adverse events. LDL-C levels decreased by about $65 \%$ after 14 days of therapy in participants receiving MK-0616.

\section{Clinical Implication}

These early phase 1 results show highly effective LDL-C lowering with MK-0616, therapy on top of statins. The safety profiles in these trials demonstrated excellent tolerability and no serious adverse events. MK-0616 single doses reduced free PCSK9 by more than 90\%, and in multiple doses, MK-0616 reduced LDL-C by 65\% after 14 days. As an oral PCSK9 inhibitor, MK-0616 has the potential to overcome barriers to treatment and allow more patients to achieve LDL-C goals and lower cardiovascular disease risk. Importantly, future studies will need to incorporate more diverse populations with more women and various race/ethnic and age groups represented. Adherence to this therapy in the long term also remains to be demonstrated. Real-world efficacy will need to be assessed given the meal-time separation required to achieve maximal results. The cost will need to be determined and may significantly limit availability. While cardiovascular outcomes have yet to be studied regarding this therapy, this orally bioavailable therapy may have significant clinically meaningful effects with future approval.

\section{Effects of aspirin on dementia and cognitive impairment in the ASCEND trial}

\section{Study Overview}

The ASCEND trial previously showed that the use of aspirin in primary prevention among patients with diabetes reduced major adverse vascular events like myocardial infarction, strokes/transient ischemic attacks (TIAs), and death from any vascular cause [18•]. However, this benefit was offset by an increase in major bleeding. Aspirin may prevent cognitive decline by a reduction in ischemic strokes and TIAs but the increase in intracranial bleeds and microbleeds may worsen cognitive impairment. Prior studies did not convincingly demonstrate the effect of aspirin in impacting dementia or cognitive decline. The ASCEND trial was a double-blind, randomized controlled trial, conducted on patients $\geq 40$ years of age with any type of diabetes and without known cardiovascular disease. A total of 15,480 participants without baseline dementia were randomized in a $2 \times 2$ factorial design to aspirin $100 \mathrm{mg}$ versus placebo (and omega-3 fatty acids $1 \mathrm{~g}$ capsule/day versus placebo). Patients were followed up for 7.4 years during the trial and 1.8 years post-trial, with $>99 \%$ followup completed for morbidity and mortality. The primary outcome of this analysis was broad dementia outcome which included reported cases of dementia, cognitive impairment, delirium/confusion, dementia medications, or referral to memory clinic or geriatric psychiatry. The narrow dementia outcome included reported cases with dementia during the study. At the final follow-up, a cognitive function test (z-score) was done based on either Telephone Interview for Cognitive Status (TICSm) and verbal fluency (VF) or the Healthy Minds test developed by the UK Biobank. The dementia outcomes were derived from hospitalizations or serious events reported by participants, the International Classification of Disease $10^{\text {th }}$ revision code diagnoses in electronic hospital admission data and death records, and other indications of cognitive impairment in follow-up and electronic records.

The mean age for participants in both the aspirin and the placebo group was 63 years. Males constituted 63\% of the participants in both groups and both groups had $94 \%$ patients with type 2 diabetes and $62 \%$ patients with hypertension. The rest of the demographic characteristics including BMI, statin use, and HBA1c were similar in both groups. Observational analyses of the risk of dementia associated with non-fatal events or major bleeds were conducted. Poisson regression analyses were done using 2-year intervals of age at risk spent with or without event. Analyses were adjusted for the number of non-dementiarelated hospitalizations $(0,1, \geq 2)$ during the interval, 
randomized treatment allocation, sex, prior diseases, and baseline predictors of dementia including a hospital diagnosis-based frailty score. Dementias that were diagnosed after a disabling stroke or intracranial bleed were excluded.

For participants who had a serious vascular events (myocardial infarction, ischemic strokes, and TIAs), there was an increase in the risk of dementia (rate ratio for the broad dementia outcome $=2.4,95 \%$ CI 1.97-2.92). For participants with major bleed, there was nearly a twofold increase in the risk of dementia (rate ratio for the broad dementia outcome $=1.96,95 \%$ CI 1.49-2.56). Revascularization did not significantly affect dementia risk (rate ratio for the broad dementia outcome $=0.91,95 \%$ CI $0.68-1.23$ ). There was a $9 \%$ non-significant reduction in the broad dementia outcome among patients on aspirin compared to placebo [aspirin 7.1\% (548/7714), placebo $7.8 \%$ (598/7713); rate ratio $0.91,95 \%$ CI 0.81-1.02)]. There was an $11 \%$ non-significant reduction in the narrow dementia outcome among patients on aspirin compared to placebo [aspirin 3.3\% (254/7714), placebo $3.7 \%$ (283/7713); rate ratio $0.89,95 \%$ CI $0.75-1.06]$. There was no statistically significant difference in the meta-analyzed cognitive score (TICSm and VF or Healthy Minds) among patients on aspirin versus placebo [mean (SE) cognitive z-score, aspirin $(n=4535): 0.004(0.015)$ versus placebo $(n=4480):-0.002(0.015)$; cognitive $\mathrm{z}$-score difference $0.012,95 \% \mathrm{CI}-0.016$ to 0.039$]$.

\section{Clinical Implication}

There was no statistically significant effect of aspirin on dementia outcomes. However, with the wide confidence intervals, the results excluded proportional harms of $>2 \%$ and benefits of $>19 \%$. In order to assess any modest proportional 15-18\% benefits of 5 to 7 years of aspirin use, larger trials, with a higher number of incident dementia cases, are required.

\section{Conclusion}

The 2021 AHA Scientific Sessions included several noteworthy trials that further the field of cardiovascular disease prevention and treatment. Overall, optimization of primary and secondary prevention is imperative in saving lives, preserving quality of life, and decreasing associated costs for patients and the healthcare system.

Notable findings regarding advancements in technologically driven remote management of CVD, lipid-lowering management therapies, and SGLT2 inhibitor use in HF reinforce the need to continually advance the field of cardiovascular medicine. With that said, further research to assess the clinical applicability of these findings is needed.

\section{Role of the Funder/Sponsor}

The Department of Veterans Affairs or the US government had no role in the design and conduct of the study; collection, management, analysis, and interpretation of the data; preparation, review, or approval of the manuscript; and decision to submit the manuscript for publication.

Funding Dr. Virani is supported by a Department of Veterans Affairs Health Services Research \& Development Service Investigator-Initiated Grants (IIR 16-072, IIR 19-069), NIH, Tahir and Jooma Family Foundation. The opinions expressed reflect those of the authors and not necessarily those of the Department of Veterans Affairs or the US government.

\section{Declarations}

Conflict of Interest Michelle T. Lee, Jerin George, Hunaina Shahab, Melody Hermel, Jamal S. Rana: None.

Salim S. Virani: Honorarium, American College of Cardiology (Associate Editor for Innovations, acc.org).

Human and Animal Rights and Informed Consent This article does not contain any studies with human or animal subjects performed by any of the authors.

\section{References}

Papers of particular interest, published recently, have been highlighted as:

- Of importance

•• Of major importance

1.• Anker SD, Butler J, Filippatos G et al. Empagliflozin in heart failure with a preservedejection fraction. N Engl J Med 2021; 385(16):1451-1461. (In a randomized trial of $\mathbf{5 9 8 8}$ heart failure patients with left ventricular ejection fraction $>40 \%$, empagliflozin (compared with placebo) was associated with a significant risk reduction in the composite endpoint of cardiovascular death and first hospitalization for heart failure.)

2. Lee MT, Mahtta D, Dlouhy L, Shahab H, Al Rifai M, Virani SS. Highlights of cardiovascular disease studies presented at the 2021 European Society of Cardiology Congress. Curr Atheroscler Rep. 2021;23(12):76.

3. Hussain A, Al Rifai M, Mahtta D, Liu J, Jain V, Virani SS. Highlights from studies presented at the American Heart Association Scientific Sessions 2020: navigating new roads in prevention. Curr Atheroscler Rep. 2021;23(1):4.

4. Jain V, Al Rifai M, Mahtta D, Liu J, Hussain A, Virani SS. Highlights from studies presented at the virtual American College of Cardiology Scientific Sessions 2021: Staying updated with the latest advancements in prevention. Curr Atheroscler Rep. 2021;23(9):50.

5. Jia X, Lee MT, Ramsey DJ et al. Facility-level variation in reported statin-associated side effects among patients with atherosclerotic cardiovascular disease-perspective from the Veterans Affair healthcare system. Cardiovasc Drugs Ther 2021; Online ahead of print. 
6. Virani SS, Kennedy KF, Akeroyd JM et al. Variation in lipidlowering therapy use in patients with low-density lipoprotein cholesterol $\geq 190 \mathrm{mg} / \mathrm{dl}$ : insights from the National Cardiovascular Data Registry-practice innovation and clinical excellence registry. Circ Cardiovasc Qual Outcomes 2018; 11(5): e004652.

7. Mcbride CL, Akeroyd JM, Ramsey DJ, et al. Statin prescription rates and their facility-level variation in patients with peripheral artery disease and ischemic cerebrovascular disease: insights from the Department of Veterans Affairs. Vasc Med. 2018;23(3):232-40.

8. Leontsinis I, Mantzouranis M, Tsioufis P, Andrikou I, Tsioufis C. Recent advances in managing primary hypertension. Fac Rev. 2020;9:4.

9. Benziger CP, Groth N. Abstract 15830: Uncontrolled and undertreated cholesterol in patients with severe hypercholesterolemia at a large healthcare system. Circulation. 2020;142(Suppl):3.

10. Bhavnani SP. Digital health: opportunities and challenges to develop the next-generation technology-enabled models of cardiovascular care. Methodist Debakey Cardiovasc J. 2020;16(4):296-303.

11. Jain V, Al Rifai M, Lee MT et al. Racial and geographic disparities in internet use in the U.S. among patients with hypertension or diabetes: implications for telehealth in the era of COVID-19. Diabetes Care 2021; 44(1): e15-7.

12. Al Rifai M, Shapiro MD, Sayani S, et al. Racial and geographic disparities in internet use in the United States among patients with atherosclerotic cardiovascular disease. Am J Cardiol. 2020;134:146-7.

13. Mahtta D, Daher M, Lee MT, Sayani S, Shishehbor M, Virani SS. Promise and perils of telehealth in the current era. Curr Cardiol Rep. 2021;23(9):115.
14. Whelton PK, Carey RM, Aronow WS et al. 2017 acc/aha/ aapa/abc/acpm/ags/apha/ash/aspc/nma/pcna guideline for the prevention, detection, evaluation, and management of high blood pressure in adults: executivesummary: a report of the American College of Cardiology/American HeartAssociation task force on clinical practice guidelines. Hypertension 2018; 71(6):1269324. (Multi-society practice guidelines provide recommendations for high blood pressure management among patients with or at risk of developing CVD.)

15. Schwartz GG, Steg PG, Szarek M, et al. Alirocumab and cardiovascular outcomes after acute coronary syndrome. N Engl J Med. 2018;379(22):2097-107.

16. Sabatine MS, Giugliano RP, Keech AC, et al. Evolocumab and clinical outcomes in patients with cardiovascular disease. N Engl J Med. 2017;376(18):1713-22.

17. Jia X, Al Rifai M, Birnbaum Y, Smith SC, Virani SS. The 2018 cholesterol management guidelines: topics in secondary ASCVD prevention clinicians need to know. Curr Atheroscler Rep. 2019;21(6):20.

18. Ascend_study_collaborative_group. Effects of aspirin for primary prevention in persons with diabetes mellitus. New England Journal of Medicine 2018; 379(16): 1529-39. (In a randomized trial of 15,480 patients with diabetes mellitus without evident cardiovascular disease, daily aspirin 100 mg (compared with placebo) was associated with decreased serious vascular event occurrence, but also caused major bleeding events.)

Publisher's Note Springer Nature remains neutral with regard to jurisdictional claims in published maps and institutional affiliations. 